\title{
REPRESENTACIÓN SOCIAL DE LOS PROBLEMAS MEDIOAMBIENTALES DEL PROFESORADO EN FORMACIÓN. APORTACIONES PARA LA EDUCACIÓN GEOGRÁFICA
}

Recepción: 22/10/2020 | Revisión: 30/11/2020 | Aceptación: 31/12/2020

\author{
José Antonio LÓPEZ FERNÁNDEZ \\ Universidad de Murcia \\ jantoniolf@um.es
}

\begin{abstract}
Resumen: Desde un enfoque interpretativo, presentamos una investigación de las representaciones sociales del profesorado en formación de educación primaria de la Universidad de Córdoba (España) sobre los problemas medioambientales. Se obtuvo la información a través de un cuestionario, que permitió la obtención de datos de tipo cuantitativo y cualitativo. A través de la representación social de las y los participantes en este estudio, se puede señalar la elevada inquietud que estos presentan respecto al estado del medioambiente. Sin embargo, son preocupantes los sesgos e imprecisiones conceptuales y la reducción del medioambiente a los hechos y sucesos naturales, resultados coincidentes con los obtenidos por otros autores en sus investigaciones. Ante esta situación, cabe reflexionar sobre las finalidades, enfoques y contenidos que se están desarrollando en la formación de los grados de magisterio de educación primaria.
\end{abstract}

Palabras clave: representación social; profesorado en formación; problemas medioambientales; educación geográfica.

\section{PRE-SERVICE TEACHERS' SOCIAL REPRESENTATION OFTHE ENVIRONMENTAL PROBLEMS. CONTRIBUTIONSTO GEOGRAPHIC EDUCATION}

\begin{abstract}
This research study revolves around the social representations of teachers in primary education training on environmental problems at the University of Córdoba (Spain). It follows an interpretative approach. Both quantitative and qualitative data were obtained through a questionnaire. Through the social representation of the participants in this study, we can point out their high concern regarding the state of the environment. However, conceptual biases and inaccuracies as well as the reduction of the environment to natural events are found, these results coinciding with those obtained in former studies. Given this situation, it is necessary to reflect on the aims, approaches and contents developed in primary school teacher training.
\end{abstract}

Keywords: social representation; trainee teachers; environmental problems; geographic education.

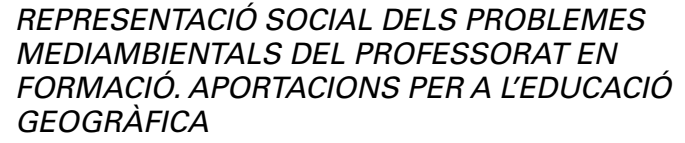

Resum: Des d'un enfocament interpretatiu, en aquest estudi plantegem una recerca sobre les representacions socials del professorat en formació d'educació primària a la Universitat de Córdoba (Espanya) sobre els problemes mediambientals. La informació es va obtenir a través d'un qüestionari amb preguntes de tipus quantitatiu $i$ qualitatiu. A través de la representació social dels subjectes participants, podem assenyalar l'elevada inquietud d'aquests respecte de lestat del medi ambient. No obstant això, preocupen els biaixos $i$ les imprecisions conceptuals, així com la reducció del medi ambient als fets i esdeveniments naturals, resultats coincidents amb els obtinguts per altres autors en investigacions anteriors. Davant d'aquesta situació, cal reflexionar sobre les finalitats, enfocaments i continguts que es desenvolupen en la formació dels graus de magisteri d'educació primària.

Paraules clau: representació social; professorat en formació; problemes mediambientals; educació geogràfica. 


\section{Introducción}

En este trabajo presentamos una investigación sobre la representación social del profesorado en formación de educación primaria sobre uno de los retos de la sociedad del siglo XXI: los problemas medioambientales. Se trata de conocer cómo los futuros profesionales conciben los fenómenos relacionados con el medioambiente y los problemas que está generando el ser humano, información que debe considerarse para su tratamiento en el aula.

El estudio se enmarca en el ámbito de la investigación interpretativa, en el que se ha construido un cuestionario que nos ha permitido obtener información mixta, tanto cuantitativa como cualitativa, con alumnado de $3^{\circ}$ curso del Grado de Educación Primaria de la Universidad de Córdoba (España) en el periodo 2017-2018.

Partimos de la base de una concepción integral y holística del medioambiente (Sauvé 2005). No obstante, el término responde a una construcción social que puede englobar diferentes significados (Calixto, 2009). La Organización de Naciones Unidas para la Educación, la Ciencia y la Cultura (UNESCO), desde finales de los años 70 del siglo XX, recoge una visión amplia del concepto de medioambiente, al contemplar que este estaría formado no solo por aspectos naturales constituidos por el agua, el aire, la temperatura, etc., sino también por aspectos sociales conformados por las relaciones del ser humano con el medio y su producción cultural (Aramburu, 2000). Desde este punto de vista, se consideran tanto los hechos y sistemas naturales como las cuestiones sociales, económicas o culturales que afectan, directa e indirectamente, al medio natural. Por tanto, ser conscientes del funcionamiento sistémico del medioambiente y el papel que tiene el ser humano es fundamental en todos los ámbitos; sobre todo cuando cada vez son más evidentes los cambios y problemas medioambientales que la sociedad provoca en el medio. Por tanto, es necesario un cambio de conciencia ambiental, que debe trabajarse como uno de los grandes retos en el ámbito educativo desde las primeras edades.

Bajo este paradigma, la investigación que presentamos se estructura en base a la representación social que muestra el profesorado en formación, para indagar en su percepción sobre las cuestiones ambientales. Así, hemos considerado los siguientes objetivos: a) Analizar las representaciones sociales sobre los problemas medioambientales que tiene el profesorado en formación de educación primaria; y b) Identificar qué tipo de cuestiones y retos ambientales son preferentes.

En este contexto, concebimos que la educación geográfica debe jugar un papel fundamental en el entendimiento de los factores y procesos ambientales. La Unión Geográfica Internacional publicó la Declaración de Lucerna sobre Educación Geográfica para el Desarrollo Sostenible (2007), donde señalaba el paradigma de la sostenibilidad para su enseñanza a todos los niveles, con la finalidad de formar una ciudadanía crítica que cuestione los pilares del desarrollo económico y la justicia social actual en todas las escalas (Benejam, 1997; De la Calle, 2013; Díaz y Felices, 2017; Fien, 1992; Pagès, 2011; Pagès y Santisteban, 2011). En este proceso, el papel del profesorado y, por tanto, su formación, es esencial. Coincidimos con otros investigadores (Claudino, Souto y Araya, 2018; Rivarosa y Perales, 2006; Souto y García, 2016) en que se trata de un campo de estudio necesario a trabajar en todos los ámbitos de la formación escolar, sobre todo desde las ciencias sociales y la educación geográfica, al permitir un tratamiento interdisciplinar y cooperativo para el desarrollo de la conciencia ambiental. 


\section{Fundamentación teórica de la investigación}

La investigación de las representaciones sociales puede aportar información relevante sobre cómo interpreta el profesorado en formación los retos actuales de la sociedad. A través de estas representaciones, se pueden evidenciar las distintas realidades concebidas por las personas, mediatizadas por sus relaciones con el entorno, con la familia y con el resto de la sociedad. Así, una parte de la investigación que se realiza desde la perspectiva crítica, con el fin de analizar los problemas relevantes del mundo o temas controvertidos (Canal, Costa y Santisteban, 2012; Legardez y Simonneaux, 2006; López-Facal, 2011; Merchán y García, 1997; Pagès y Santisteban, 2011; Prieto y Lorda, 2011 y 2012), parte del análisis de las representaciones sociales del profesorado en formación, ya que permiten conocer el punto de partida a través del cual poder construir o reconstruir el conocimiento, así como forjar capacidades y competencias docentes (Materán, 2008).

El concepto de representación social surge a mediados del siglo XIX, desarrollado posteriormente por el psicólogo Sergei Moscovici, a partir de la teoría construida por Durkheim sobre la representación colectiva. Siguiendo a Moscovici (1979), Villarroel (2007) considera que la representación social es una reconstrucción del conocimiento común, en el que intervienen el desarrollo cognoscitivo, simbólico y cultural en el que se mueven y crecen las personas. Para Jodelet (1984), las representaciones sociales se presentan bajo formas muy variadas, compuestas por imágenes, sistemas de referencia que nos permiten dar una interpretación de lo que nos sucede. Para esta psicóloga francesa, discípula de Moscovici, el concepto de representación social se identifica con el conocimiento espontáneo e ingenuo, denominado habitualmente como conocimiento común y que es complementario al pensamiento científico. Se puede contemplar, pues, como un modelo de pensamiento que se recibe y se transmite a través de la tradición, la educación y el conocimiento social.

Vemos perfectamente que siempre se trata de lo mismo. A saber: una manera de interpretar y de pensar nuestra realidad cotidiana, una forma de conocimiento social. Y correlativamente, la actividad mental desplegada por individuos y grupos a fin de fijar una posición en relación con sistemas, acontecimientos, objetos y comunicaciones que los conciernen. [...] Antes que nada, concierne a la manera cómo nosotros, sujetos sociales, aprehendemos los acontecimientos de la vida diaria, las características de nuestro medio ambiente, las informaciones que en él circulan, a las personas de nuestro entorno próximo o lejano. (Jodelet, 1984:473)

Las representaciones sociales también han sido cuestión de análisis respecto a diferentes temáticas socioambientales (Materán, 2008; Olmos, González-Monfot y Pagès, 2017; Sant, Casas y Pagès, 2011; Souto y García, 2016). En esta línea, Bertorello y Hurtado (2018: 64) entienden que el análisis sobre el conocimiento que presenta el alumnado debe «partir de aquellas representaciones o conocimientos previos que los estudiantes... poseen». Souto y García (2016:187), desde la didáctica de la Geografía, señalan que las representaciones sociales «surgen cuando los individuos debaten temas de interés mutuo o cuando existe el eco de los acontecimientos seleccionados como significativos...». En este sentido, la educación geográfica ha jugado y debe jugar un papel importante para afrontar los retos ambientales que suceden a diferentes escalas.

Es necesario contribuir, desde la educación geográfica, a edificar una sociedad más solidaria y equitativa en el largo plazo. Una sociedad sustentable, en la cual los jóvenes de hoy, adultos del mañana, tengan suficientes conocimientos, elementos de juicio y actitudes que les permitan tomar decisiones adecuadas con respecto al espacio geográfico. (Araya, 2010:69). 
El estudio de Bertorello y Hurtado (2018) destaca la falta de incorporación del enfoque crítico en las propuestas de aula y el predominio de un paradigma tradicional y memorístico en el profesorado en formación. Calixto $(2008 ; 2012)$ analiza las representaciones del medioambiente en un grupo de futuros maestros y maestras en la ciudad de México, confeccionando según los resultados, cinco categorías de representaciones: antropocéntricas, utilitaristas, culturales, naturalistas y globalizantes, resultando sobresalientes las primeras. Las representaciones sociales del profesorado en formación sobre el medioambiente han sido utilizadas, entre otros, por Morales, Caurín y Souto (2013), cuyos resultados son fundamentales para establecer las secuencias y propuestas didácticas encaminadas a mejorar la formación docente.

Resulta, pues, de interés seguir investigando las representaciones sociales sobre el medioambiente desde la perspectiva de la educación geográfica ya que, como señala Calixto (2009), la introducción de los problemas medioambientales en la escuela presenta un reduccionismo teórico importante, al recibir mayor importancia los conceptos ecológicos y naturales, muy por encima de los de carácter social, donde intervienen una serie de elementos como las creencias como parte del sentido común; las opiniones; las percepciones como procesos de clasificación de la realidad; y las concepciones como marcos de referencia cognitiva construidos por las personas. Por tanto, la educación geográfica puede resultar indispensable por el carácter de síntesis e interdisciplinar que la caracteriza, para la comprensión de los problemas medioambientales, siempre y cuando se aleje del mero conocimiento enciclopédico, y se acerque a postulados críticos que puedan dar respuesta a los problemas de «nuestro entorno social» (De la Calle, 2013:35).

\section{Metodología}

Este trabajo se encuadra en el marco de la investigación interpretativa, un enfoque metodológico en el que intentamos relacionar teoría y práctica, a partir del estudio analítico de lo que está ocurriendo en un ámbito concreto (Coller, 2005; Stake, 1999). Con este planteamiento metodológico, pretendemos obtener resultados a partir de un cuestionario en el que ha participado profesorado en formación de educación primaria, e intervenir posteriormente en una siguiente fase para desarrollar la «capacidad de reflexión crítica [...] analizar su propio contexto y realidad cotidiana, y tomen sus propias decisiones sobre las acciones que más les conviene realizar para hacer frente a sus limitaciones» (Martínez, 2007:33). Esta finalidad es imprescindible si, desde el ámbito educativo, se quiere concienciar y actuar ante cuestiones medioambientales (Young, 1993).

Los datos obtenidos son de tipo mixto, tanto cualitativos como cuantitativos. Consideramos que los resultados de este tipo de investigación pueden ayudar en la toma de decisiones y otorgar más información en los procesos de autoreflexión en el ámbito educativo, así como replantear la finalidad de las propuestas didácticas que se vienen realizando en la formación universitaria para hacer frente a los retos que hoy presenta el mundo y que derivan en numerosos problemas sociales (Bisquerra et al., 2004). 


\section{1. Ámbito de la investigación}

La investigación se ha llevado a cabo en la Facultad de Ciencias de la Educación de la Universidad de Córdoba durante el curso 2017-2018; es decir, con grupos que presentan unas características determinadas, y cuyos resultados pueden facilitar la comparación con otros ejemplos. La muestra de la población seleccionada para participar en la investigación han sido los cuatro grupos de tercer curso (Tabla 1). La heterogeneidad de las personas participantes responde a la realización del cuestionario en un momento determinado del año en curso, previo a tratar los contenidos relacionados con la educación geográfica y ambiental. Mientras que en los grupos 1 y 2 se tomaron datos de dos de los tres subgrupos medianos, en los grupos 3 y 4 solo participó uno de ellos. Se trata, pues, de una muestra no probabilística e intencional.

\begin{tabular}{|c|c|c|c|c|c|c|c|c|}
\hline \multicolumn{2}{|c|}{ Grupo 1 (G1) } & \multicolumn{2}{c|}{ Grupo 2 (G2) } & \multicolumn{2}{c|}{ Grupo 3 (G3) } & \multicolumn{2}{c|}{ Grupo 4 (G4) } & Total \\
\hline H & M & H & M & H & M & H & M & 102 \\
\hline 9 & 28 & 12 & 25 & 6 & 7 & 5 & 13 & \\
\hline
\end{tabular}

Tabla 1. Muestra de participantes en la investigación. Fuente: Elaboración propia.

Es un periodo formativo interesante para analizar las representaciones sociales sobre temáticas medioambientales, donde entre otras asignaturas, se cursan Didáctica de las Ciencias Sociales y Didáctica de Ciencias Experimentales, antes de que en el curso siguiente se desarrolle la asignatura obligatoria Didáctica del Medioambiente. La toma de datos en un periodo inicial pretende que, según los resultados, se puedan planificar y aplicar diversas actuaciones en la mejora de la formación docente. A la población objeto de estudio se les presuponen ciertos conocimientos y valores, ya establecidos en parte durante la educación obligatoria, que influirán de un modo u otro en su concepción del medioambiente y en sus futuras intervenciones didácticas.

El total de cuestionarios realizados fue de 105, desglosados por grupos y sexo según se observa en la Tabla 1. No obstante, tras el tratamiento de los datos en una revisión inicial, se rechazaron tres cuestionarios por su falta de respuestas y concreción $(\mathrm{N}=102)$. A cada cuestionario se le asignó para su análisis un identificador que corresponde con el grupo al que pertenece más el número correspondiente $(\mathrm{G} 1-1, \ldots, \mathrm{G} 2-1, \ldots)$

\subsection{Instrumento para la recogida de información y criterios para categorizar los resultados}

Para la recogida de información, se desarrolló un cuestionario ad hoc que, en una fase inicial, se pasó a un grupo control de 5 personas para observar su funcionalidad y operatividad. La temática y la línea de la investigación, descriptiva e interpretativa, además de las características y el número de la muestra objeto de estudio, son los factores que determinaron la selección de esta herramienta (Walker, 1989). En la construcción del instrumento se disponen las preguntas 1 y 2 para recoger 
variables de tipo cualitativo y carácter abierto. Estas cuestiones tienen la finalidad de explorar la concepción del profesorado en formación sobre los problemas medioambientales, lo que requiere del análisis previo del contenido de las respuestas para poder proceder a su posterior categorización. Mientras, las cuestiones 3 y 4 predisponen una variable cerrada de índole cuantitativa a través de una escala Likert (la reproducción fiel de las preguntas se recoge en la Tabla 2).

De este modo, en la primera pregunta se cuestiona directamente por los retos actuales que debe afrontar la sociedad, con la intención de averiguar la concepción del medioambiente y si los temas ambientales se consideran como reto de la sociedad. Este interrogante también nos permite indagar qué cuestiones se contemplan a escala local o global, un aspecto que también hemos contemplado a través de la pregunta 2 (Zenovi, 2006). En esta se utilizó una imagen concreta. Las imágenes son un instrumento de gran valor a la hora de analizar las representaciones sociales. En este caso, corresponde a la ciudad de Madrid, con la evidencia de una boina de contaminación. Ante situaciones de estabilidad climática y la presencia de altas presiones, Madrid constituye un paradigma de contaminación ambiental en España como consecuencia de la polución procedente del transporte por carretera, la industria y aparatos de aire acondicionado. Este tipo de situaciones son reflejadas por los medios de comunicación numerosas veces al año. En concreto, la fotografía mostrada al profesorado en formación, cuya fuente corresponde a la Agencia EFE, fue publicada por el diario Público con motivo de los elevados niveles de ozono el 30 de junio de 2016.

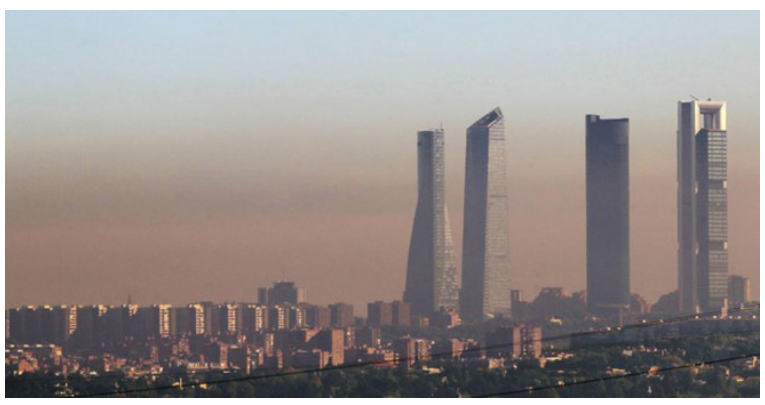

Figura 1. Vista de la ciudad de Madrid durante un periodo de contaminación. Fuente: Diario Público (EFE).

En la pregunta 3, la categorización se realizó en función de la importancia que reciben diferentes situaciones ambientales, acompañada de una justificación cualitativa, seleccionándose en este trabajo las situaciones de sequía en España y el mundo, la contaminación ambiental de las ciudades y las catástrofes humanas con origen natural, como inundaciones, terremotos o tsunamis. En la pregunta $n^{\circ} 4$ la categorización se determina por el grado de preocupación personal sobre los problemas medioambientales, contemplándose: 1, ninguna preocupación; 2, poca preocupación; 3 bastante y 4 mucho. La intención de este interrogante, de carácter cuantitativo y formulado al final del cuestionario, se presenta con la intención de comparar los resultados con los obtenidos en la primera pregunta. 


\begin{tabular}{|l|l|}
\hline \multicolumn{1}{|c|}{ Cuestiones solicitadas } & \multicolumn{1}{c|}{ Criterios planteados para su análisis } \\
\hline $\begin{array}{l}\text { 1. En tu opinión, ¿cuáles son los } \\
\text { retos que debe afrontar la sociedad } \\
\text { actual? }\end{array}$ & $\begin{array}{l}\text { Representación social de los problemas medioambientales. } \\
\text { Consideración de temáticas medioambientales como reto actual de } \\
\text { la sociedad.Y atención a la escala de acción (global o local) }\end{array}$ \\
\hline $\begin{array}{l}\text { 2. Observa la siguiente imagen. } \\
\text { Realiza una descripción de la misma } \\
\text { y reflexiona sobre los diferentes } \\
\text { tipos de problemas que se dan en } \\
\text { este lugar }\end{array}$ & $\begin{array}{l}\text { Interpretación de las consecuencias de los problemas } \\
\text { medioambientales según su escala: global y/o local. }\end{array}$ \\
\hline $\begin{array}{l}\text { 3. Exposición de una serie de } \\
\text { problemas medioambientales. } \\
\text { Valóralos según la importancia que elementos visibles y no visibles } \\
\text { le darías a cada uno }\end{array}$ & $\begin{array}{l}\text { Consideración o grado de importancia de los problemas } \\
\text { planteados, causas y consecuencias a nivel medioambiental a }\end{array}$ \\
\hline $\begin{array}{l}\text { 4. Indica tu grado de preocupación escala Likert (1-4) } \\
\text { respecto a los problemas } \\
\text { medioambientales del mundo } \\
\text { actual }\end{array}$ & $\begin{array}{l}\text { Grado de importancia por la situación ambiental global del planeta } \\
\text { a través de una escala Likert (1-4) }\end{array}$ \\
\hline
\end{tabular}

Tabla 2. Categorización de los resultados según las preguntas planteadas en el cuestionario Fuente: Elaboración propia.

Los criterios para clasificar y categorizar los resultados se establecieron a posteriori; es decir, una vez revisadas las respuestas de las personas participantes (Tabla 2). Esta disposición nos sitúa en el uso de un modelo inductivo, a través del análisis del contenido de las respuestas obtenidas, sobre todo en las cuestiones 1 y 2 . Los criterios seguidos para la categorización de los resultados responden a conceptos medioambientales, sociales y espaciales y, sobre todo, a la concepción sistémica y relacional del medioambiente.

El planteamiento o dimensión sobre la que se construyó el cuestionario y, por tanto, sobre la que se vertebra la investigación es la representación social que tiene el profesorado en formación de educación primaria sobre los problemas medioambientales. Dentro de esta dimensión general, hemos querido prestar atención a la concepción que tienen los futuros docentes respecto a los retos ambientales para la sociedad; cómo perciben la incidencia de los diferentes problemas según la escala espacial de incidencia; y la influencia que, para ellas y ellos, tiene el ser humano en su interacción con el medio.

La metodología seguida para el análisis de las preguntas se basa en el conteo de conceptos aparecidos en las respuestas, lo que nos proporciona la frecuencia de los términos utilizados y, por tanto, su grado de importancia. Este método, utilizado en otras investigaciones (Abric, 2001; Conde y Armas, 2019), permite generar datos cuantitativos y cualitativos para reflejar los puntos nodales y complementarios de la representación social de las personas. Así, intentamos analizar el sentido común que predomina en el profesorado en formación sobre problemas medioambientales; un sentido común que se estructura en base al conocimiento científico que presentan los individuos, la influencia del saber popular y los medios de comunicación, etc. 


\section{Resultados}

En la primera pregunta destaca la importancia que reciben los temas medioambientales, al señalarse conceptos concretos o problemáticas ambientales actuales. A grandes rasgos, la muestra objeto de estudio realiza una distinción entre retos sociales, económicos y ambientales, si bien no destaca la relación multidireccional y sistémica existente entre ellos, planteándolos como situaciones diferenciadas e inconexas. Entre los problemas de tipo social, resaltan situaciones como la desigualdad, la falta de valores como la tolerancia o el respeto a otras formas de pensar, la influencia de las redes sociales, problemas relacionados con las migraciones, etc.

G3-1. «Uno de los retos más importantes que se debe afrontar consistiría en la creación y formación de valores morales positivos, haciendo consciente a la sociedad de los numerosos problemas que ocurren diariamente en nuestro entorno...También deberíamos hacer conscientes a la sociedad de los problemas que ocurren a gran escala relacionados con la mala gestión del poder, la inmigración, el racismo, la falta de solidaridad,...».

G3-2. «La sociedad actual tiene una carencia de valores muy grande. Se pierden los valores del respeto hacia las personas y el entorno que nos rodea, además de otros como la tolerancia y la igualdad... Otros problemas son la igualdad de género, la contaminación, la sobreexplotación de recursos...».

\begin{tabular}{|l|c|c|c|c|}
\hline $\begin{array}{l}\text { Principales conceptos de carácter } \\
\text { medioambiental }\end{array}$ & $\begin{array}{c}\text { Referencias } \\
\text { GR. 1 }\end{array}$ & $\begin{array}{c}\text { Referencias } \\
\text { GR. 2 }\end{array}$ & $\begin{array}{c}\text { Referencias } \\
\text { GR. 3 }\end{array}$ & $\begin{array}{c}\text { Referencias } \\
\text { GR. 4 }\end{array}$ \\
\hline Contaminación & 15 & 16 & 5 & 9 \\
\hline Cambio climático & 11 & 6 & 2 & 3 \\
\hline Medioambiente & 11 & 14 & 4 & 0 \\
\hline Escasez de agua & 0 & 6 & 0 & 0 \\
\hline Desarrollo sostenible & 6 & 0 & 0 & 2 \\
\hline Consumismo & 0 & 4 & 1 & 2 \\
\hline
\end{tabular}

Tabla 3. Referencias a problemas medioambientales identificados en la pregunta 1. ¿Cuáles son los retos que debe afrontar la sociedad actual? Fuente: Elaboración propia.

Entre los restos económicos, sobresale la crisis económica y el paro. Respecto a problemas de tipo ambiental, son numerosos los discursos que reflejan preocupación por la contaminación ( como «la naturaleza») y las consecuencias del cambio climático, pero sin llegar a considerarlos como un problema social (Tabla 3 ).

Por el contrario, en la pregunta 4 de nuestro cuestionario, que interroga directamente por el grado de preocupación de los problemas medioambientales (que hemos planteado para comparar con la valoración del primer interrogante), los resultados señalan que más $90 \%$ del alumnado participante se muestra bastante o muy preocupado por la situación ambiental en general, mientras que el $10 \%$ restante se muestra algo preocupado. Vemos, por tanto, que al cuestionarles directamente por su grado de preocupación medioambiental éste resulta muy elevado, pero si se les pregunta por los retos actuales de la sociedad, en muchos casos los problemas medioambientales no son tenidos en cuenta. 
La pregunta 1 también nos permite diferenciar cómo se perciben determinadas situaciones ambientales según la escala a la que se producen (Gomera, 2011) (Tabla 4). Así, la casi totalidad de las evidencias ambientales se refieren a cuestiones de ámbito global (Zenovi, 2006), destacando conceptos como la contaminación, la protección del medioambiente y el cambio climático. Este resultado es casi idéntico, proporcionalmente, en todos los grupos.

\begin{tabular}{|c|c|c|c|c|c|c|c|c|}
\hline VARIABLES & $\begin{array}{c}\text { Ref. } \\
\text { G1 }\end{array}$ & $\%$ & $\begin{array}{c}\text { Ref. } \\
\text { G2 }\end{array}$ & $\%$ & $\begin{array}{c}\text { Ref. } \\
\text { G3 }\end{array}$ & $\%$ & $\begin{array}{c}\text { Ref. } \\
\text { G4 }\end{array}$ & $\%$ \\
\hline Retos globales & 50 & 89,3 & 58 & 81,7 & 17 & 85 & 22 & 75,9 \\
\hline Retos locales & 0 & 0 & 4 & 5,6 & 0 & 0 & 4 & 13,8 \\
\hline No perciben p. m. & 6 & 10,7 & 9 & 12,7 & 3 & 15 & 3 & 10,3 \\
\hline Total & 56 & 100 & 71 & 100 & 20 & 100 & 29 & 100 \\
\hline
\end{tabular}

Tabla 4. Distribución de referencias ambientales según problemas globales o locales. Fuente: Elaboración propia.

Entre las hipótesis de esta imagen sobre el medioambiente, además de un conocimiento ambiguo y parcial, puede inferirse la influencia que ejerce la información sensacionalista de los medios de comunicación, ya sea en televisión, prensa y, sobre todo, por las redes sociales, cargadas con la búsqueda de un impacto mediático en el ámbito global (Gomera, 2011).

Observamos, por tanto, el predominio de cuestiones problemáticas de índole global, muy por encima de problemas medioambientales de carácter local. Ejemplo de un enfoque global se muestra en la siguiente exposición:

G1-1. «Hambre, aumento de desigualdades entre las clases sociales, cambio climático, agotamiento de recursos, contaminación, corrupción y falta de confianza en el poder político, vulneración de derechos humanos y despreocupación o falta de consecuencias ante tales injusticias, falta de puestos de trabajo, aumento descontrolado de la población, falta de cuidado y consideración por el medioambiente o progresiva despreocupación por el bienestar social, lo importante es el rendimiento de la persona, no su felicidad».

G4-9. «En mi opinión, algunos de los retos que debe afrontar la sociedad actual son: la contaminación existente en todo el mundo y todos los problemas que ello conlleva».

Mientras que respuestas con carácter más local, o que requieren de actuaciones a nivel individual, las encontramos en G2-10 o G2-11:

G2-10. «Ahorrar en el consumo de electricidad y de agua. En el caso de la electricidad, buscar y adoptar medidas para gastar la menos posibles, y en el caso del agua, no malgastarla».

G2-11. «Cuidado del medioambiente, evitar la contaminación acústica, hacer uso del transporte público o el tema del reciclaje».

La segunda pregunta propone la observación de una imagen, que presenta un ámbito marcadamente urbano como la ciudad de Madrid. Se pide al estudiantado realizar una descripción y reflexionar sobre los diferentes tipos de problemas que observan. El objetivo de esta pregunta es analizar qué tipo de problemas sobresalen sobre un ámbito urbano.

En primer lugar, en el análisis global de esta cuestión destaca la relación que establece el 
profesorado en formación entre el concepto de ciudad y los problemas que ésta genera. Para ellos, la ciudad constituye un foco de contaminación, de sobreexplotación de recursos y de transformación del entorno, al considerar que la construcción en altura con grandes edificios, la existencia de la industria y las dinámicas de la movilidad, conllevan numerosos problemas relacionados con el entorno natural y que estos, a su vez, tienen consecuencias sobre la salud de las personas (Tabla 5).

\begin{tabular}{|c|c|c|c|c|c|c|c|}
\hline \multicolumn{2}{|l|}{ GRUPO 1} & \multicolumn{2}{|l|}{ GRUPO 2} & \multicolumn{2}{|l|}{ GRUPO 3} & \multicolumn{2}{|l|}{ GRUPO 4} \\
\hline CONCEPTOS & $N^{o}$ & CONCEPTOS & $N^{o}$ & CONCEPTOS & $N^{o}$ & CONCEPTOS & $N^{o}$ \\
\hline $\begin{array}{l}\text { Contaminación } \\
\text { ambiental }\end{array}$ & 23 & Contaminación & 23 & $\begin{array}{l}\text { Contaminación } \\
\text { ambiental }\end{array}$ & 13 & $\begin{array}{l}\text { Contaminación } \\
\text { ambiental }\end{array}$ & 19 \\
\hline Contaminación & 17 & $\begin{array}{l}\text { Contaminación } \\
\text { ambiental }\end{array}$ & 15 & Masificación & 4 & Superpoblación & 8 \\
\hline $\begin{array}{l}\text { Contaminación } \\
\text { acústica }\end{array}$ & 8 & $\begin{array}{l}\text { Contaminación } \\
\text { acústica }\end{array}$ & 8 & $\begin{array}{l}\text { Destrucción } \\
\text { espacio natural }\end{array}$ & 2 & $\begin{array}{l}\text { Explotación de } \\
\text { recursos }\end{array}$ & 3 \\
\hline Sobrepoblación & 7 & $\begin{array}{l}\text { Problemas de } \\
\text { salud }\end{array}$ & 7 & $\begin{array}{l}\text { Contaminación } \\
\text { aire }\end{array}$ & 2 & $\begin{array}{l}\text { Contaminación } \\
\text { visual }\end{array}$ & 1 \\
\hline $\begin{array}{l}\text { Problemas de } \\
\text { salud }\end{array}$ & 5 & $\begin{array}{l}\text { Reducción } \\
\text { espacios verdes }\end{array}$ & 6 & $\begin{array}{l}\text { Contaminación } \\
\text { lumínica }\end{array}$ & 2 & Enfermedades & 1 \\
\hline $\begin{array}{l}\text { Contaminación } \\
\text { lumínica }\end{array}$ & 3 & Masificación & 6 & $\begin{array}{l}\text { Contaminación } \\
\text { acústica }\end{array}$ & 2 & $\begin{array}{l}\text { Calentamiento } \\
\text { global }\end{array}$ & 1 \\
\hline $\begin{array}{l}\text { Desequilibrio } \\
\text { MA y ser } \\
\text { humano }\end{array}$ & 2 & $\begin{array}{l}\text { Sobreexplotación } \\
\text { de recursos }\end{array}$ & 4 & $\begin{array}{l}\text { Consumo } \\
\text { energía }\end{array}$ & 1 & $\begin{array}{l}\text { Desigualdades } \\
\text { sociales }\end{array}$ & 1 \\
\hline $\begin{array}{l}\text { Calentamiento } \\
\text { global }\end{array}$ & 1 & $\begin{array}{l}\text { Contaminación } \\
\text { lumínica }\end{array}$ & 4 & Impacto visual & 1 & $\begin{array}{l}\text { Problemas de } \\
\text { movilidad }\end{array}$ & 2 \\
\hline $\begin{array}{l}\text { Impacto } \\
\text { ambiental }\end{array}$ & 1 & Consumismo & 3 & $\begin{array}{l}\text { Contaminación } \\
\text { de ríos }\end{array}$ & 1 & $\begin{array}{l}\text { Contaminación } \\
\text { acústica }\end{array}$ & 3 \\
\hline $\begin{array}{l}\text { Escasez de } \\
\text { recursos }\end{array}$ & 1 & Deforestación & 2 & $\begin{array}{l}\text { Poder } \\
\text { económico }\end{array}$ & 1 & $\begin{array}{l}\text { Ansiedad y } \\
\text { estrés }\end{array}$ & 2 \\
\hline Deforestación & 1 & Exceso de tráfico & 2 & $\begin{array}{l}\text { Desigualdades } \\
\text { sociales }\end{array}$ & 1 & Depresión & 1 \\
\hline Consumismo & 1 & $\begin{array}{l}\text { Contaminación } \\
\text { del agua }\end{array}$ & 1 & Estrés & 2 & $\begin{array}{l}\text { Problemas de } \\
\text { nutrición }\end{array}$ & 1 \\
\hline & & $\begin{array}{l}\text { Invasión de } \\
\text { espacios } \\
\text { naturales }\end{array}$ & 1 & $\begin{array}{l}\text { Calentamiento } \\
\text { global }\end{array}$ & 1 & & \\
\hline & & $\begin{array}{l}\text { Impacto } \\
\text { ambiental }\end{array}$ & 1 & Desempleo & 1 & & \\
\hline & & $\begin{array}{l}\text { Calentamiento } \\
\text { global }\end{array}$ & 1 & $\begin{array}{l}\text { Problemas de } \\
\text { movilidad }\end{array}$ & 2 & & \\
\hline & & $\begin{array}{l}\text { Rotura capa de } \\
\text { Ozono }\end{array}$ & 1 & & & & \\
\hline & & $\begin{array}{l}\text { Problemas de } \\
\text { movilidad }\end{array}$ & 1 & & & & \\
\hline & & Basura & 1 & & & & \\
\hline
\end{tabular}

Tabla 5. Frecuencia de señalización de conceptos relacionados con los problemas medioambientales en un entorno urbano. Fuente: Elaboración propia. 
Observamos el predominio de conceptos como contaminación, identificándose como principal problema de la ciudad, aunque la mayor parte lo sigue haciendo de forma ambigua, sin especificar el tipo de contaminación.

Aunque son los menos, otros relatos introducen mayor cantidad de conceptos y diferentes temáticas medioambientales, como el calentamiento global, el consumo de energía, la contaminación del agua y la rotura de la capa de ozono. Además, este tipo de discursos, más concretos a la hora de señalar los conceptos naturales, inciden en la influencia de la sociedad en la generación de problemas medioambientales y sus efectos sobre la población.

G2-1. «En segundo lugar también se dan problemas de contaminación que pueden ser de muchos tipos: contaminación acústica provocada por actividades económicas que se realizan en la ciudad, así como la causada por los distintos medios de transporte, contaminación del agua por todos los residuos que se generan y se vierten en los ríos».

G2-14. «La alta concentración de la población ocasiona problemas de movilidad y de gran contaminación producida por los medios de transporte, principalmente los coches».

Respecto a los problemas de tipo social o humano, el profesorado en formación es capaz de identificar una amplia variedad de problemas sociales, como la generación de ruidos, mayor tráfico y dificultades de movilidad, la producción de basuras, enfermedades como la ansiedad, el estrés, la depresión y otros problemas de salud, pero su representatividad es menor respecto al conjunto de hechos naturales. Es decir, son solo algunos participantes los que hacen alusión a este tipo de cuestiones relacionadas con el entorno urbano.

En la tercera pregunta se plantean una serie de situaciones ambientales para que el alumnado realice una valoración escalar, en función de la importancia que le otorgan. El objetivo de esta pregunta es analizar cómo se categorizan y valoran las situaciones propuestas y de qué modo justifican su respuesta. No obstante, debido a la complejidad de los datos y para el interés de nuestra investigación, hemos optado por analizar tres de los supuestos planteados, relacionados con procesos naturales que pueden ser inducidos por el ser humano y que provocan importantes consecuencias medioambientales: la 2, situaciones de sequía en España y el mundo; la 5, la contaminación ambiental de las ciudades; la 7, las situaciones de catástrofes humanas con origen natural, como inundaciones, terremotos o tsunamis.

Sobre la sequía y sus consecuencias, una amplia mayoría considera que se trata de un problema bastante o muy significativo. Sin embargo, aunque su importancia porcentual es menor, en los cuatro grupos aparecen valoraciones de que se trata de una situación poco representativa, que se puede solventar con la tecnología actual (Tabla 6):

G1-3. «La sequía española no me parece tan grave porque tenemos recursos, pero en los países menos desarrollados es un asunto grave».

G1-7. «Con los avances actuales supone un problema menor». 


\begin{tabular}{|c|c|c|c|c|c|c|c|c|}
\hline & $\mathbf{1 *}^{*}$ & $\mathbf{\%}$ & $\mathbf{2}^{*}$ & $\mathbf{\%}$ & $\mathbf{3}^{*}$ & $\mathbf{\%}$ & $\mathbf{4}^{*}$ & $\mathbf{\%}$ \\
\hline GRUPO 1 & 0 & 0 & 4 & 11,11 & 10 & 27,78 & 22 & 61,11 \\
\hline GRUPO 2 & 0 & 0 & 2 & 5,41 & 9 & 24,32 & 26 & 70,27 \\
\hline GRUPO 3 & 0 & 0 & 1 & 7,69 & 4 & 30,77 & 8 & 61,54 \\
\hline GRUPO 4 & 0 & 0 & 1 & 5,56 & 1 & 5,56 & 16 & 88,89 \\
\hline
\end{tabular}

*1. Nada significativo; 2. Poco significativo; 3. Bastante significativo; 4. Muy significativo. Tabla 6. Respuestas del profesorado en formación sobre los problemas de sequía (supuesto 2) que afectan a la población de España y del mundo. Fuente: Elaboración propia.

Este tipo de justificaciones ante el problema planteado denotan la consideración de una visión distorsionada y parcial de la realidad. En primer lugar, no conciben la sequía como un hecho de interés en España, a pesar de la controversia social que se evidencia en los medios de comunicación. Y tampoco señalan o conciben la sequía como uno de los mayores problemas medioambientales en diferentes regiones del mundo. Por otro, algunos discursos muestran plena confianza en los avances tecnológicos para mitigar este tipo de situaciones, característica principal de la visión utilitarista del medio.

Respecto al supuesto n 5, según la Tabla 7, destaca el elevado porcentaje de participantes que consideran los problemas de contaminación en las ciudades como un problema bastante y muy significativo; la totalidad en el caso del grupo 4. Estos resultados se muestran en consonancia con la información obtenida en la pregunta 2. No obstante, no faltan opiniones en los grupos 1, 2 y 3 que perciben que se trata de una situación poco significativa.

\begin{tabular}{|c|c|c|c|c|c|c|c|c|}
\hline & $\mathbf{1 *}^{*}$ & $\mathbf{\%}$ & $\mathbf{2}^{*}$ & $\mathbf{\%}$ & $\mathbf{3}^{*}$ & $\mathbf{\%}$ & $\mathbf{4}^{*}$ & $\mathbf{\%}$ \\
\hline GRUPO 1 & 0 & 0 & 3 & 8,33 & 12 & 33,33 & 21 & 58,33 \\
\hline GRUPO 2 & 0 & 0 & 2 & 5,41 & 16 & 43,24 & 19 & 51,35 \\
\hline GRUPO 3 & 0 & 0 & 2 & 5,41 & 16 & 43,24 & 19 & 51,35 \\
\hline GRUPO 4 & 0 & 0 & 0 & 0 & 6 & 33,33 & 12 & 66,67 \\
\hline
\end{tabular}

*1. Nada significativo; 2. Poco significativo; 3. Bastante significativo; 4. Muy significativo. Tabla 7. Respuestas del profesorado en formación sobre los problemas de contaminación producida en las ciudades debido al uso de coches, aparatos eléctricos, etc. (supuesto 5). Fuente: Elaboración propia.

En cuanto a los problemas generados por sucesos naturales (inundaciones, terremotos, tsunamis), en la Tabla 8 aparece cierta diversidad en las respuestas obtenidas. En los grupos 2 y 3 no llegan al 70\% las personas que entienden que se trata de un problema bastante o muy significativo. En el 2, el 32\% de estudiantes indican que es una cuestión poco o nada importante, justificando en algún caso, incluso, la poca utilidad de las ciencias sociales para aportar soluciones ante este tipo de situaciones ambientales.

G1-6. «El planeta tiene sus ritmos y sus ciclos. Es un riesgo que ha de asumir el ser humano porque es inevitable y pueden ser nocivas. Pero suelen ser aislados. Creo que es poco significativo porque el estudio de las ciencias sociales poco puede hacer...»

G2-8. «Debido a que ocurren en pocas ocasiones, y pienso que el ser humano no tiene la culpa de ello». G2-10. «Es importante, pero algo que no está directamente en nuestras manos». 


\begin{tabular}{|c|c|c|c|c|c|c|c|c|}
\hline & $\mathbf{1}^{*}$ & $\mathbf{\%}$ & $\mathbf{2}^{*}$ & $\mathbf{\%}$ & $\mathbf{3}^{*}$ & $\mathbf{\%}$ & $\mathbf{4}^{*}$ & $\mathbf{\%}$ \\
\hline GRUPO 1 & 0 & 0 & 7 & 19,44 & 11 & 30,56 & 18 & 50,00 \\
\hline GRUPO 2 & 2 & 5,41 & 10 & 27,03 & 17 & 45,95 & 8 & 21,62 \\
\hline GRUPO 3 & 0 & 0 & 4 & 30,77 & 4 & 30,77 & 5 & 38,46 \\
\hline GRUPO 4 & 0 & 0 & 2 & 11,11 & 9 & 50,0 & 8 & 44,44 \\
\hline
\end{tabular}

*1. Nada significativo; 2. Poco significativo; 3. Bastante significativo; 4 . Muy significativo. Tabla 8. Respuestas del profesorado en formación sobre los problemas provocados por sucesos naturales como inundaciones o tsunamis (supuesto 7). Fuente: Elaboración propia.

En cierto modo, ponen de manifiesto desconocer el papel que juega el ser humano cuando ocurren ciertas «catástrofes naturales». No exponen, por tanto, las consecuencias de las acciones antrópicas derivadas de una mala ocupación del territorio y es que, parte de lo que la sociedad estima como una situación de catástrofe natural, presenta como causa fundamental una mala planificación urbana, el uso indebido de los llanos de inundación o de las costas, actuaciones que tienen como consecuencia la destrucción de espacios y, en ocasiones, la pérdida de vidas humanas. Al contrario, la mayor parte de los discursos justifican que son hechos a tener en cuenta, pero que están fuera del alcance del ser humano, a merced de los sucesos naturales.

\section{Discusión de los resultados}

La información recogida refleja que el profesorado en formación de educación primaria concibe una elevada preocupación por los temas medioambientales, al considerarlos como un reto social en la actualidad y para el futuro. En mayor parte, los describe como uno de los principales problemas que debe afrontar la sociedad; situación que se evidencia a partir de la pregunta 4, de índole cuantitativa. Sin embargo, a través de la pregunta 1 (que se formula de forma abierta) es reseñable que más del 20\% no refleje los problemas medioambientales como reto actual. Mientras tanto, quien sí los contempla, incide en que los problemas medioambientales son aquellos derivados de la contaminación, que afectan a la destrucción del entorno y la naturaleza, siendo escasamente significativo quien establece una relación sistémica, donde se considere a la sociedad como factor principal en el desencadenamiento de muchos problemas medioambientales.

A través de la pregunta 1, también podemos destacar la importancia significativa que reciben los problemas ambientales de carácter global. Por el contrario, es una evidencia la falta de conocimiento de los sucesos cercanos. Las narrativas del profesorado en formación hablan de procesos ambientales como la contaminación, la protección del medioambiente y el cambio climático; mientras que es escasamente representativa la relación a hechos cercanos. De los 102 cuestionarios, resulta complicado encontrar problemas ambientales específicos de su entorno cercano. Esta percepción de la realidad puede corresponder directamente con una menor preocupación por los sucesos a escala local y, por tanto, un menor grado de participación en su medio más próximo. Situación que pone de manifiesto el concepto de hipermetropía ambiental, caracterizado por la valoración de los problemas medioambientales de ámbito global que, sin embargo, obvian las 
cuestiones locales (Gomera, 2011; Uzzell, 2000).

$\mathrm{Al}$ cuestionarles por los problemas medioambientales de los entornos urbanos (pregunta 2), las situaciones predominantes en las respuestas vuelven a ser la contaminación ambiental y la destrucción del entorno. Por su parte, se refleja una amplia variedad de problemas sociales, muchos de ellos derivados de la actual relación con el entorno, pero corresponden a la identificación de determinadas personas por lo que no podemos destacar que se trate de un conocimiento o concepción generalizado entre los participantes.

Tras el análisis de las tres primeras preguntas, podemos resaltar de igual modo que las representaciones del profesorado muestran una evidente ambigüedad conceptual. A través de las respuestas a las cuestiones 1, 2 y, sobre todo en los supuestos planteados en la pregunta 3, observamos que existe una generalizada falta de precisión conceptual a la hora de concretar los tipos de contaminación; no se mencionan problemas globales como la presencia de plásticos en los océanos; es casi inexistente la falta de consideración sistémica del medioambiente, siendo poco valoradas las acciones humanas y sus consecuencias sobre los diferentes espacios; predomina una visión antropocéntrica, por un lado, y eurocéntrica, por otro, con escasa atención a muchos problemas medioambientales que existen en otras partes del mundo. En este sentido, Morales, Caurín y Souto (2013) ya constataron una visión deformada de la realidad social y ambiental del planeta, con «unas concepciones previas, en el alumnado, llenas de estereotipos y aprendizajes fragmentados y desvirtuados en detrimento del conocimiento integral y global» (p. 104).

Las representaciones sociales de los problemas medioambientales obtenidas en nuestro estudio pueden estructurarse según la categorización realizada por Calixto (2008) a partir de los estudios de Reigota (1990). Este último realizó una clasificación de las concepciones del medio entre naturalistas, globalizantes y antropocéntricas. A partir de esta tipificación, Calixto planteó cinco categorías de representaciones: naturalistas (donde predominan términos como el agua, y el medioambiente se entiende como naturaleza), globalizantes (con discursos donde predominan interrelaciones entre lo natural y lo social), antropocéntricas utilitarias (uso masivo de términos relacionados con las condiciones de vida de las personas y la consideración de un medio supeditado a las necesidades de la sociedad), antropocéntricas pactuadas (se es consciente de la relación histórica entre el ser humano y la naturaleza, pero los bienes naturales son percibidos en función de los intereses de las personas) y antropocéntricas culturales (donde se da importancia al legado cultural de la sociedad en su proceso de adaptación al medio). En nuestro caso, se dan con claridad discursos naturalistas, donde el medioambiente se entiende como naturaleza y donde el ser humano se le presupone la capacidad de modificarlo en su beneficio (Calixto, 2008) pero sin reducirlo a simple recurso. Menos representativo es en nuestro estudio el grupo que podríamos considerar globalizante, ya que son poco numerosos los cuestionarios donde se observe la percepción de la relación sistémica del medioambiente. Por el contrario, son abundantes las concepciones de carácter antropocéntrico según las diferentes variedades distinguidas por Calixto. En ellas predomina una concepción de supremacía del ser humano respecto al medio, donde la naturaleza está al servicio de este y la tecnología es un elemento indispensable para ejercer su control.

Toda esta información debe tenerse en cuenta ya que, como señala Calixto (2009), la re- 
presentación social pone de manifiesto la toma de decisiones cotidianas, relacionadas, por ejemplo, con la forma de planificar las finalidades, objetivos y contenidos de la educación ambiental que posteriormente se llevan a cabo en las aulas (López y Oller, 2018).

En este sentido, debido al carácter ambiental que se le presupone a la educación geográfica, deberían revisarse las finalidades de la enseñanza de la geografía en la educación obligatoria, así como en la formación del futuro profesorado, dejando atrás los postulados tradicionales, marcadamente descriptivos y escasamente operativos en el desarrollo de las capacidades y competencias para una ciudadanía consciente respecto al impacto global y local que la sociedad está generando en el mundo (Bertorello y Hurtado, 2018).

Desde hace tiempo, la investigación e innovación relacionada con la enseñanza de las ciencias sociales y de la geografía apuesta por la inclusión en las aulas de los problemas socioambientales que ocurren en el mundo, a pesar de las reticencias y la dificultad por derrocar el predominio del modelo tradicional, basado en su mayoría por la memorización de los libros de texto (García Pérez y De Alba, 2008). En este sentido, cabe considerar si el profesorado en formación tiene los conocimientos y capacidades para, en este caso, abordar con solvencia los problemas medioambientales en educación primaria. Coincidimos, por tanto, con lo reflejado por MorenoCrespo y Moreno-Fernández (2015:90) que indican que «se hace necesario que las facultades de formación del profesorado y de Ciencias de la Educación renueven el compromiso que tienen con la sociedad en general y con sus estudiantes en particular».

Cebrián y Junyent (2014: 45) ya resaltaron, al respecto, la carencia de capacidades del futuro profesorado por su falta para «visionar diferentes escenarios de futuro, la interdisciplinariedad, la gestión de las emociones o el fomento del pensamiento crítico». Una situación identificada por Álvarez-García, Sureda-Negre y Comas-Forgas (2018:127 y 128), al señalar que el profesorado en formación «carece de los conocimientos ambientales deseables para ejercer su práctica docente y un pobre compromiso de acción en la resolución de la problemática ambiental».

\section{Conclusiones}

La investigación realizada nos ha posibilitado la consecución de los objetivos propuestos, relacionados con analizar la representación del profesorado en formación de educación primaria sobre los problemas medioambientales. E, identificar, por otro, los sesgos y carencias que muestran al respecto.

El profesorado en formación inicial de educación primaria percibe los temas ambientales como un reto actual de la sociedad. Sin embargo, hemos evidenciado el conocimiento impreciso que presentan sobre los procesos ambientales y el papel que otorgan al ser humano. Es notoria la visión antropocéntrica a través de sus respuestas y podemos señalar el predominio de la concepción reduccionista que tienen respecto al medioambiente; una concepción en la que puede haber intervenido la educación geográfica recibida (Binimelis y Ordinas, 2016).

También hemos podido identificar el protagonismo que reciben los procesos ambientales a nivel global; mientras que son escasamente percibidos los sucesos locales, lo que puede 
determinar la participación en entornos cercanos y su participación en la solución de problemas (Zenovi, 2006).

A pesar de las limitaciones de este tipo de investigación (momento y ámbito concreto, la posible mejora del instrumento para la toma de datos, así como el análisis de los resultados), sería necesario considerar la imagen que tiene el futuro profesorado sobre los problemas medioambientales, de cara a revisar la efectividad de la enseñanza durante la educación obligatoria así como en el periodo de formación de maestras y maestros, analizándose el tipo de enseñanzas que presuponen disciplinas como la Geografía (De Miguel, 2018).

\section{Agradecimientos}

A los revisores anónimos, por sus acertados comentarios y valiosas sugerencias. A la profesora Montserrat Oller Freixa, por colaborar y contribuir en la formación del autor.

\section{Referencias bibliográficas}

Abric, J. C. (2001). Prácticas sociales y representaciones. México: Coyoacán.

Alcira, A., y Perales, F. J. (2006). La resolución de problemas ambientales en la escuela y la formación inicial de maestros. Revista Iberoamericana de Educación, 40, 111-124. doi: https://doi.org/10.35362/rie400785

Álvarez-García, O., Sureda-Negre, J., y Comas-Forgas, R. (2018). Evaluación de las competencias ambientales del profesorado de primaria en formación inicial: estudio de caso. Enseñanza de las Ciencias, 36, 117-141. doi: https://doi.org/10.5565/rev/ensciencias.2338

Aramburu, F. (2000). Medio ambiente y educación. Madrid, Editorial Síntesis

Benejam, P. (1997). La selección y secuenciación de los contenidos sociales. En P. Benejam y J. Pagès (Coord.), Enseñar y aprender Ciencias Sociales, Geografía e Historia (pp. 71-95). Barcelona, ICE Universidad de Barcelona/Horsori.

Bertorello, S. E., y Hurtado, E. J. (2018). Los problemas socio-ambientales en la región sur de Córdoba abordados desde las ciencias sociales para el profesorado de educación primaria. Revista de Investigación en Didáctica de las Ciencias Sociales. REIDICS, 2, 55-69. doi: https://doi.org/10.17398/2531-0968.02.55

Binimelis, J., y Ordinas, A. (2016). Los conocimientos mínimos de Geografía en los estudios de Grado en Educación Primaria. Revista Complutense de Educación, 2(3), 1309-1326. https://doi.org/10.5209/rev_RCED.2016.v27.n3.48586

Bisquerra, R. (Coord.) (2004). Metodología de la investigación educativa. Madrid: Editorial La Muralla S. A.

Calixto, R. (2008). Representaciones sociales del medio ambiente. Perfiles educativos, 120, 33-62. Recuperado de: https://www.iisue.unam.mx/perfiles/articulo/2008-120-representacionessociales-del-medio-ambiente.pdf 
Calixto, R. (2009). Representaciones sociales del medio ambiente. México: Universidad Pedagógica Nacional.

Calixto, R. (2012). Investigación en educación ambiental. Revista Mexicana de Educación Educativa, 17, 1019-1033. Recuperado de: http://www.comie.org.mx/revista/v2018/rmie/ index.php/nrmie/article/view/341

Canal, M., Costa, D., y Santisteban, A. (2012). El alumnado ante problemas sociales relevantes: ¿cómo los interpreta? ¿Cómo piensa la participación? En N. de Alba, F. F. García y A. Santisteban (Eds.), Educar para la participación ciudadana en la enseñanza de las Ciencias Sociales (pp. 391-400). Sevilla, AUPDCS.

Cebrian, G., y Junyent, M. (2014). Competencias profesionales en Educación para la Sostenibilidad: un estudio exploratorio de la visión de futuros maestros. Enseñanza de las ciencias, 32, 2949. doi: https://doi.org/10.5565/rev/ensciencias. 877

Claudino, S., Souto, X. M., y Araya, F. (2018). Los problemas socio-ambientales en Geografía. Una lectura iberoamericana. Revista Lusófona de Educação, 39, 55-73. doi: https://10.24140/ issn.1645-7250.rle39.04

Coller, X. (2005). Estudios de casos. Cuadernos metodológicos 30. Madrid: Centro de investigaciones sociológicas.

Conde, J., y Armas, X. (2019). Representaciones sociales del alumnado de educación primaria sobre el patrimonio. Estudio por medio de la asociación de palabras. Revista Interuniversitaria de Formación del Profesorado, 94(33,2), 187-201. doi: https://doi.org/10.47553/rifop. v33i2.73266

De la Calle, M. (2013). La enseñanza de la Geografía ante los nuevos desafíos ambientales, sociales y territoriales. En. R. de Miguel, M.L. de Lázaro y M. J. Marrón (Eds.), Innovación en la enseñanza de la Geografía ante los desafíos sociales y territoriales. (pp. 33-52). Zaragoza, España: Institución Fernando el Católico.

De Miguel, R. (2018). Geografía y tiempo contemporáneo: educación geográfica y enseñanza de las ciencias sociales para el mundo global. Revista de Investigación en Didáctica de las Ciencias Sociales. REIDICS, 2, 36-54. doi: https://doi.org/10.17398/2531-0968.02.36

Díaz, N., y Felices, $M^{a}$. M. (2017). Problemas sociales relevantes en el aula de primaria: la "cartografía de la controversia" como método. Revista de Investigación en Didáctica de las Ciencias Sociales, REIDICS, 1, 24-38. doi: https://doi.org/10.17398/2531-0968.01.24

Fien, J. (1992). Geografía, sociedad y vida cotidiana. Documents d'Anàlisi Geogràfica, 21, 73-90. Recuperado de: https://www.raco.cat/index.php/DocumentsAnalisi/article/view/41559

García Pérez, F. F., y De Alba, N. (2008). ¿Puede la escuela del siglo XXI educar a los ciudadanos y ciudadanas del siglo XXI?. Scripta Nova. Revista Electrónica de Geografía y Ciencias Sociales, vol. XII, No. 270 (122), 1-15.

Gomera, A. (2011). Análisis, medición y distribución de la conciencia ambiental en el alumnado universitario: una herramienta para la educación ambiental. (Tesis doctoral). Universidad de Córdoba, Córdoba. 
Jodelet, D. (1984). La representación social: fenómenos, concepto y teoría. En S. Moscovici (Ed.), Psicología Social (pp. 469-494). Barcelona: Paidos.

Legardez, A., y Simonneaux, L. (2006). L'école à l'épreuve de l'actualité. Enseigner les questions vives. París: Esf.

López-Facal, R. (2011). Conflictos sociales candentes en el aula. En J. Pagès y A. Santisteban. Les qüestions socialment vives $i$ l'ensenyament de les ciències socials (pp. 65-76). Barcelona: Servei de Publicacions de la Universitat Autònoma de Barcelona. Col. Documents, 97.

López-Fernández, J. A., y Oller, M. (2018). Los problemas medioambientales en la formación del profesorado de educación primaria. Revista de Investigación en Didáctica de las Ciencias Sociales. REIDICS, 4, 93-109. doi: https://doi.org/10.17398/2531-0968.04.93

Martínez González, R. (2007). La investigación en la práctica educativa: Guía metodológica de investigación para el diagnóstico y evaluación en los centros docentes. Madrid: Ministerio de Educación y Ciencia.

Materán, A. (2008). Las representaciones sociales: un referente teórico para la investigación educativa. Geoenseñanza, 13(2), 243-248. Recuperado de: http://www.saber.ula.ve/ handle/123456789/28953

Merchán, F. J., y García, F. F. (1997). El tratamiento de los problemas de nuestro mundo en la enseñanza obligatoria. Aula de innovación educativa, 61, 9-12.

Morales, A. J., Caurín, C., y Souto, X. M. (2013). Percepción del mundo: mapas mentales y problemas socioambientales. Didáctica Geográfica, 14, 91-108. Recuperado de: http://www.agegeografia.es/didacticageografica/index.php/didacticageografica/article/view/262

Moreno-Crespo, P., y Moreno-Fernández, O. (2015). Problemas socio-ambientales: concepciones del profesorado en formación inicial. Andamios, 12(19), 73-96.

Moscovici, S. (1979 [1961]). El psicoanálisis, su imagen y su público. Huemul S.A., Buenos Aires.

Olmos, R., González-Monfort, N., y Pagès, J. (2017). Las representaciones sociales del alumnado sobre la crisis. ¿Qué soluciones ofrece el alumnado ante los problemas económicos? Didáctica de las Ciencias Experimentales y Sociales, 32, 51-70. doi: https://10.7203/ DCES.32.9394

Pagès, J. (2011). ¿Qué se necesita saber y saber hacer para enseñar Ciencias Sociales? La didáctica de las Ciencias Sociales y la formación de maestros y maestras. Edetania, 40, 67-81. Recuperado de https://revistas.ucv.es/index.php/Edetania/article/view/276

Pagès, J., y Oller, M. (2007). Las representaciones sociales del derecho, la justicia y la ley de un grupo de adolescentes catalanes de $4^{\circ}$ de ESO. Enseñanza de las ciencias sociales. Revista de Investigación, 6, 3-19. Recuperado de :https://www.raco.cat/index.php/EnsenanzaCS/ article/view/126325

Pagès, J. y Santisteban, A. (2011). Les qüestions socialment vives i lensenyament de les ciències socials. Barcelona: Servei de Publicacions de la Universitat Autònoma de Barcelona. Col. Documents, 97.

Prieto, M. N., y Lorda, M. A. (2011). Los problemas sociales o temas de controversia social en la enseñanza de la Geografía. Aportes para su aplicación en el aula. Revista Geográfica 
de América Central, 47, 1-18. Recuperado de: https://www.revistas.una.ac.cr/index.php/ geografica/article/view/2828

Prieto, M. N., y Lorda, M. A. (2012). Propuesta metodológica para la enseñanza de la problemática medioambiental como contenido programático en la educación geográfica. Revista Universitaria de Geografía, 21, 11-30. Recuperado de: https://www.redalyc.org/articulo. oa? $\mathrm{id}=383239104001$

Reigota, M. (1990). Les représentations sociales de lenvironnement et les pratiques pédagogiques quotidiennes des professeurs de Sciences a São Paulo-Brésil (Tesis de doctorado en Pedagogía de la Biología). Universidad Católica de Lovaina, Lovaina.

Rivarosa, A., y Perales, F. J. (2006). La resolución de problemas ambientales en la escuela y en la formación inicial de maestros. Revista Iberoamericana de Educación, 40, 111-124. doi: https://doi.org/10.35362/rie400785

Sant, E., Casas, M. T., y Pagès, J. (2011). Participar para aprender la democracia. Las representaciones sociales de jóvenes catalanes sobre la participación democrática. Uni-pluri/versidad Vol. 11, 2. Universidad de Antioquia. Medellín. Col. Recuperado de: http://aprendeenlinea. udea.edu.co/revistas/index.php/unip/issue/current

Sauvé, L. (2005). Uma cartografia das corrientes em educaçao ambiental (pp. 17-46). In Sato, M. et Carvalho, I. (Dir.), Educação ambiental - Pesquisa e desafios. Porto Alegre: Artmed

Souto, X. M., y García, D. (2016). La geografía escolar ante el espejo de su representación social. Didáctica Geográfica, 17, 177-201. Recuperado de: http://www.age-geografia.es/ didacticageografica/index.php/didacticageografica/article/view/365

Stake, R. E. (1998). Investigación con estudio de caso. Madrid: Morata

Unión Geográfica Internacional (2007). Declaración de Lucerna sobre Educación Geográfica para el Desarrollo Sostenible. Comisión de Educación Geográfica de la Unión Geográfica Internacional. Pekín. Recuperado de: http://www.igu-cge.org/Charters-pdf/2016/ IGU_2016_spanish.pdf

Uzzell, D. L. (2000).The psycho-spatial dimensionof global environmental problems. Journal of Environmental Psychology, 20, 307-318

Villarroel, G. (2007). Las representaciones sociales: una nueva relación entre el individuo y la sociedad. Fermentun: Revista venezolana de Sociología y Antropología, 49, 434-454. Recuperado de https://www.redalyc.org/pdf/705/70504911.pdf

Walker, R. (1989). Métodos de investigación para el profesorado. Madrid: Morata.

Young, R. (1993). Teoría crítica de la educación y discurso en el aula. Madrid: Paidós.

Zenovi, V. (2006). Geografía, Problemáticas ambientales a diferentes escalas. Gobierno de la Ciudad de Buenos Aires: Ministerio de Educación. 